Tôhoku Math. Journ.

28 (1976), 311-326.

\title{
INFLECTION-FREE EMBEDDINGS OF GRASSMANN MANIFOLDS
}

\author{
HIROSHI ÔIKE
}

(Received May 20, 1974)

0. Introduction. In [8] and [3], W. F. Pohl and E. A. Feldman have considered higher order tangent bundles of a smooth manifold $M$ and the higher order nondegenerate immersion of $M$ into Euclidean spaces. In [9], [10], [11] and [12], H. Suzuki obtained some higher order nonimmersion theorems of projective spaces into Euclidean spaces or projective spaces by means of characteristic classes, $\gamma$-operations and spin operations.

In [14], C. Yoshioka obtained complete formulas of Stiefel-Whitney classes of higher order tangent bundles of complex projective spaces and Dold manifolds and he applied his results to higher order non-immersions of these spaces. In [7], the complete formulas of higher order tangent bundles of complex projective spaces and lens spaces in $K O$-rings of these spaces and the complete formulas of Stiefel-Whitney classes of higher order tangent bundles of quaternion projective spaces are obtained. In [5], J. A. Little studied singularities of submanifolds of higher dimentional Euclidean spaces and he obtained many valuable results. In this paper, we show that the well known embeddings of Grassmann manifolds in hyperplane of Euclidean space of all hermitian matrices are inflectionfree, i.e., second order nondegenerate. Our result is a generalization of J. A. Little's result on real projective spaces (Theorem 3.7 of [5]).

This paper is divided into three sections. In the first section we give descriptions of osculating space, inflection point, inflection-free immersion and first order normal form of immersion and we show some examples on Klein bottle, the second section contains the main result and its proof and in the final section we give an alternative proof of the Theorem 1.1 in [4] and a remark on the Lemma 4.1 in the same paper.

1. Preliminaries. Let $X: M^{n} \rightarrow \boldsymbol{R}^{N}$ be an immersion of a smooth manifold of dimension $n$ into a Euclidean space of dimension $N$. Let $p \in M^{n}$, and $u_{1}, \cdots, u_{n}$ local coordinates valid in a neighborhood of $p$, with $u_{1}=\cdots=u_{n}=0$ at $p$. 
Let

$$
X_{i}=\left(\frac{\partial X}{\partial u_{i}}\right)_{p}, \quad X_{i j}=\left(\frac{\partial^{2} X}{\partial u_{i} \partial u_{j}}\right)_{p},
$$

the derivatives being evaluated at $p$. By the (second order) osculating space of $X$ at $p$ we mean the linear space through $p$ spanned by the $X_{i}$ and $X_{i j}$. If the osculating space at $p$ has dimension $(1 / 2) n(n+1)+n$ or $N$, the maximum possible, we say that $X$ is second order nondegenerate at $p$, and if not so, we say that $p$ is an inflection point (first order inflection point). It is not hard to see that these notions are independent of the choice of the local coordinates $u_{1}, \cdots, u_{n}$. We say that $X$ is inflection-free or second order nondegenerate, if $X$ is second order nondegenerate at every $p$ in $M^{n}$. Let $T_{p}$ and $N_{p}$ denote respectively the tangent and normal spaces of $X$ at $p$. For any vector $v$ in $\boldsymbol{R}^{N}$ let $v^{\perp}$ denote the orthogonal projection of $v$ into $N_{p}$. Now let $x(t)$ be a curve on $M^{n}$ such that $x(0)=p$. Then the orthogonal projection into $N_{p}$ of the second derivative of $X(x(t))$ at 0 depends only on the first derivative of $X(x(t))$ at 0 , as is well known (at least in the case of curve on surface in ordinary space, and as is proved in the same way in higher dimensions). Thus we have a map $\nu_{p}: T_{p} \rightarrow N_{p}$ which assigns to each $v \in T_{p}$ the orthogonal projection in $N_{p}$ of the second derivative vector of a curve on $M^{n}$ through $p$ whose tangent vector at $p$ is $v$. We say that $\nu_{p}$ is the first order normal form or the second fundamental form of $X$ at $p$. To find an analytic expression for $\nu_{p}$ we consider the curves

$$
u_{i}=x_{i} t, \quad 1 \leqq i \leqq n \text {. }
$$

Then

$$
X^{\prime}(0)=\sum_{i=1}^{n} x_{i} X_{i}, \quad \text { and } \quad X^{\prime \prime}(0)^{\perp}=\sum_{i=1}^{n} \sum_{j=1}^{n} x_{i} x_{j} X_{i j}^{\perp}
$$

Hence

$$
\nu_{p}\left(\sum_{i} x_{i} X_{i}\right)=\sum_{i} x_{i}^{2} X_{i i}^{\perp}+2 \sum_{i<j} x_{i} x_{j} X_{i j}^{\perp} .
$$

$\nu_{p}$ is a vector-valued quadratic form. Then we may regard $\nu_{p}$ as a symmetric bilinear form defined on $T_{p}$, valued in $N_{p}$, i.e., $\nu_{p}: O^{2}\left(T_{p}\right) \rightarrow N_{p}$ homomorphism, where $O^{2}\left(T_{p}\right)$ is 2-fold symmetric tensor product of $T_{p}$, as follows

$$
\nu_{p}\left(X_{i} \bigcirc X_{j}\right)=X_{i j}^{\perp} \text { for } X_{i} \bigcirc X_{j} \in O^{2}\left(T_{p}\right) \text {. }
$$

$X$ is second order nondegenerate at $p$ if and only if $\nu_{p}: O^{2}\left(T_{p}\right) \rightarrow N_{p}$ is injective or surjective. If we regard $\nu_{p}: T_{p} \rightarrow N_{p}$ as a vector-valued 
quadratic form, $X$ is second order nondegenerate at $p$ if and only if linear subspace generated by $\nu_{p}\left(T_{p}\right)$ is of dimension $(1 / 2) n(n+1)$ or equal to $N_{p}$.

Now we give some examples on Klein bottle. Consider the equivalence relation in $\boldsymbol{R}^{2}$ given by

$$
\left(\Theta+2 \pi m,(-1)^{m} \theta+2 \pi n\right) \sim(\Theta, \theta) \text { for } m, n=0, \pm 1, \pm 2, \cdots .
$$

The quotient space $K$ under this relation is Klein bottle. For the fixed real numbers $R, r$ such that $0<r<R$, let $X: R^{2} \rightarrow R^{4}, X(\Theta, \theta)=$ $(x, y, u, v)$ be as follows.

$$
\begin{aligned}
x & =(R+r \cos \theta) \cos \Theta & y & =(R+r \cos \theta) \sin \Theta, \\
u & =r \sin \theta \cos \frac{\Theta}{2}, & v & =r \sin \theta \sin \frac{\Theta}{2} .
\end{aligned}
$$

Then $X$ induces a smooth embedding of $K$ in $\boldsymbol{R}^{4}$. The following map $Y: \boldsymbol{R}^{2} \rightarrow \boldsymbol{R}^{4}$ induces also a smooth embedding of $K$ in $\boldsymbol{R}^{4}$. For the fixed real numbers $R, r$ such that $0<r<R$, let $Y(\Theta, \theta)=(x, y, u, v)$ be as follows

$$
\begin{aligned}
x & =\left(R+r \sin \theta \sin \frac{\Theta}{2}\right) \cos \Theta, & y & =\left(R+r \sin \theta \sin \frac{\Theta}{2}\right) \sin \Theta, \\
u & =r \sin \theta \cos \frac{\Theta}{2}, & v & =r \cos \theta .
\end{aligned}
$$

Set $X\left(\boldsymbol{R}^{2}\right)=K^{*}$ and $Y\left(\boldsymbol{R}^{2}\right)=K^{* *} . \quad X, Y$ are universal covering maps over $K^{*}, K^{* *}$ respectively.

The set of all inflection points of $K^{*}$ is a simple closed curve on $K^{*}$ such that

$$
\left\{R \cos \alpha\left(\begin{array}{l}
\cos \alpha \cos \Theta \\
\cos \alpha \sin \Theta \\
\sin \alpha \cos \frac{\Theta}{2} \\
\sin \alpha \sin \frac{\Theta}{2}
\end{array}\right) \in R^{4} ; 0 \leqq \Theta<4 \pi\right\},
$$

where $\sin \alpha=r / R(0<\alpha<\pi / 2)$. The set of all inflection points of $K^{* *}$ is a set of two points of $K^{* *}$ as follows

$$
\{(-R+r, 0,0,0),(-R-r, 0,0,0)\} .
$$

Next we give an inflection-free immersion of $K$ in $R^{4}$. For the fixed real numbers $0<a, 0<b$, let $Z: \boldsymbol{R}^{2} \rightarrow \boldsymbol{R}^{4}, Z(\Theta, \theta)=(x, y, u, v)$ be as follows 


$$
\begin{array}{ll}
x=a \cos \theta \cos \Theta & y=a \cos \theta \sin \Theta \\
u=b \sin \theta \cos \frac{\Theta}{2} & v=b \sin \theta \sin \frac{\Theta}{2} .
\end{array}
$$

Then the following proposition is easily seen by brief calculations.

Proposition 1.1. The above $Z: \boldsymbol{R}^{2} \rightarrow \boldsymbol{R}^{4}$ induces an inflection free immersion of $K$ in $\boldsymbol{R}^{4}$.

It is easily seen that the second order tangent bundle of $K$ is trivial. Thus characteristic classes, $\gamma$-operations or spin operations do not serve to study inflection-free non-immersion or non-embedding of $K$. The author does not know if there is an inflection-free embedding of Klein bottle $K$ in $\boldsymbol{R}^{4}$ or not.

2. Inflection-free embeddings of Grassmann manifolds. For the rest of this paper, $\boldsymbol{F}$ will denote the field $\boldsymbol{R}$ of real numbers, the field $\boldsymbol{C}$ of complex numbers or the field $\boldsymbol{H}$ of quaternions. In natural way, $\boldsymbol{R} \subset \boldsymbol{C} \subset \boldsymbol{H}$. Let $\boldsymbol{F}^{n}$ be the right $\boldsymbol{F}$-vector space of all $n$-dimensional column vectors and let $G L(n ; F)$ be the $n$-dimensional general linear group over $\boldsymbol{F}$. Denote by $M(n, m ; \boldsymbol{F})$ the right vector space of all $n \times m$-matrices over $\boldsymbol{F}$. Then $M(n, m ; \boldsymbol{F})$ is $m n$-dimensional over $\boldsymbol{F} . \quad *: M(n, m ; \boldsymbol{F}) \rightarrow$ $M\left(m, n ; \boldsymbol{F}^{\prime}\right)$ will denote the adjoint operator, i.e., for $A \in M(n, m ; \boldsymbol{F})$, $A^{*} \in M(m, n ; \boldsymbol{F})$ is the transposed-conjugate matrix of $A$. The right inner product over $\boldsymbol{F}$ for $M(n, m ; \boldsymbol{F})$ is defined as $(A, B)=\operatorname{tr}\left(A^{*} B\right)$ for $A, B \in M(n, m ; \boldsymbol{F})$. Set $M(n ; \boldsymbol{F})=M(n, n ; \boldsymbol{F})$ and denote by $E_{n}$ the identity matrix of $M(n ; \boldsymbol{F})$. Now for $x \in \boldsymbol{F}, \operatorname{Re} x$ will denote the real part of $x$. Then $\operatorname{Re} x=(1 / 2)(x+\bar{x})$ for $x \in F, \operatorname{Re}(y x)=\operatorname{Re}(x y)$, i.e., $y x+\bar{x} \bar{y}=x y+\bar{y} \bar{x}$ for $x, y \in F$. By these facts, we have the following lemma.

\section{LEMMA 2.1.}

$$
\begin{array}{lll}
\operatorname{Re} \operatorname{tr}(X)=\frac{1}{2} \operatorname{tr}\left(X+X^{*}\right)=\operatorname{Re} \operatorname{tr}\left(X^{*}\right) & \text { for } \quad X \in M(n ; \boldsymbol{F}), \\
\operatorname{Re} \operatorname{tr}(Y X)=\operatorname{Re} \operatorname{tr}(X Y) & \text { for } \quad X, Y \in M(n ; \boldsymbol{F}), \\
\operatorname{Re} \operatorname{tr}\left(A B^{*}\right)=\operatorname{Re} \operatorname{tr}\left(A^{*} B\right), i . e ., & \\
\operatorname{tr}\left(A B^{*}+B A^{*}\right)=\operatorname{tr}\left(A^{*} B+B^{*} A\right) & \text { for } \quad A, B \in M\left(n, m ; \boldsymbol{F}^{\prime}\right) .
\end{array}
$$

Let $U(n ; \boldsymbol{F})$ be the group of all unitary matrices of degree $n$ over $\boldsymbol{F}$, i.e., $U\left(n ; \boldsymbol{F}^{\prime}\right)=\left\{U \in M\left(n ; \boldsymbol{F}^{\prime}\right) ; U^{*} U=E_{n}\right\}$. Then $U(n ; \boldsymbol{R})=O(n)$, $U(n ; \boldsymbol{C})=U(n)$ and $U(n ; \boldsymbol{H})=S p(n)$, in standard notations. $H(n ; \boldsymbol{F})$ will denote the $\boldsymbol{R}$-vector space of all hermitian matrices over $\boldsymbol{F}$, i.e., $H\left(n ; \boldsymbol{F}^{\prime}\right)=\left\{X \in M\left(n ; \boldsymbol{F}^{\prime}\right) ; X^{*}=X\right\}$.

$H(n ; \boldsymbol{F})$ is of dimension $(1 / 2) d_{F} n(n-1)+n$ over $\boldsymbol{R}$, where $d_{F}=1,2$ 
or 4 for $\boldsymbol{F}=\boldsymbol{R}, \boldsymbol{C}$ or $\boldsymbol{H}$ respectively. The inner product for $H(n ; \boldsymbol{F})$ over $\boldsymbol{R}$ is defined by $(X, Y)=\operatorname{Re} \operatorname{tr}(X Y)$ for $X, Y \in H(n ; \boldsymbol{F})$. Set $H_{\mu}\left(n ; \boldsymbol{F}^{\prime}\right)=\left\{X \in H\left(n ; \boldsymbol{F}^{\prime}\right) ; \operatorname{tr}(X)=\mu\right\}$, for $\mu \in \boldsymbol{R}$. Then $H_{\mu}\left(n ; \boldsymbol{F}^{\prime}\right)$ is regarded as a hyperplane in the Euclidean space $H(n ; F)$. Let $U(n ; F)$ act linearly on $H(n ; \boldsymbol{F})$ in obvious manner: $U(X)=U X U^{*}, \quad U \in U(n ; \boldsymbol{F})$ and $X \in H(n ; \boldsymbol{F})$. The following lemma is easily seen.

LEMmA 2.2. The action of $U(n ; F)$ preserves inner product of $H(n ; \boldsymbol{F})$ and trace of each matrix of $H(n ; \boldsymbol{F})$. Therefore $H_{\mu}\left(n ; \boldsymbol{F}^{\prime}\right)$ for $\mu \in \boldsymbol{R}$ is invariant under the action of it.

The following lemma is well known [13].

LEMmA 2.3. For each $X \in H(n ; \boldsymbol{F})$, there exists a $U \in U(n ; \boldsymbol{F})$ such that $U^{*} X U$ is a real diagonal matrix and the set of all eigenvalues of a hermitian matrix is unique for the matrix.

For $0 \leqq m \leqq n, M_{n, m}^{*}\left(F^{\prime}\right)$ will denote the set of all orthogonal projections of $F^{n}$ of rank $m$, i.e.,

$$
M_{n, m}^{*}(\boldsymbol{F})=\left\{P \in M\left(n ; \boldsymbol{F}^{\prime}\right) ; P^{2}=P, P^{*}=P \quad \text { and } \quad \operatorname{tr}(P)=m\right\} .
$$

Then $M_{n, m}^{*}(\boldsymbol{F}) \subset H_{m}\left(n ; \boldsymbol{F}^{\prime}\right)$. The following lemma is easily seen.

LEMma 2.4. For $P \in M(n ; \boldsymbol{F})$, the following statements are equivalent:

(i) $P \in M_{n, m}^{*}(F)$,

(ii) there exists a $U \in U(n ; \boldsymbol{F})$ such that

$$
P=U\left(\begin{array}{cc}
E_{m} & 0 \\
0 & 0
\end{array}\right) U^{*} \text {. }
$$

Thus the action of $U(n ; \boldsymbol{F})$ on $H\left(n ; F^{\prime}\right)$ is transitive on $M_{n, m}^{*}(\boldsymbol{F})$.

For $0<m \leqq n$, set

$V_{n, m}\left(F^{\prime}\right)=\left\{A \in M(n, m ; \boldsymbol{F}) ; A^{*} A=E_{m}\right\}$,

$V_{n, m}^{\prime}\left(\boldsymbol{F}^{\prime}\right)=\left\{A \in M\left(n, m ; \boldsymbol{F}^{\prime}\right) ; A^{*} A \in G L\left(m ; \boldsymbol{F}^{\prime}\right)\right\}$,

$M_{n, m}\left(\boldsymbol{F}^{\prime}\right)$; the set of all $m$-dimensional $\boldsymbol{F}$-subspaces of $\boldsymbol{F}^{n}$.

$V_{n, m}(\boldsymbol{F})$ is the Stiefel manifold over $\boldsymbol{F}, V_{n, m}^{\prime}(\boldsymbol{F})$ is the set of all $n \times m$-matrices over $\boldsymbol{F}$ of rank $m$ and $M_{n, m}(\boldsymbol{F})$ is the Grassmann manifold over $\boldsymbol{F} . \quad \pi: V_{n, m}^{\prime}\left(\boldsymbol{F}^{\prime}\right) \rightarrow M_{n, m}\left(\boldsymbol{F}^{\prime}\right)$ will denote the natural projection, i.e., for $A \in V_{n, m}^{\prime}\left(F^{\prime}\right), \pi(A) \in M_{n, m}\left(F^{\prime}\right)$ is the subspace spanned by $m$ column vectors of $A$. Consider the following map $p: V_{n, m}^{\prime}(\boldsymbol{F}) \rightarrow H(n ; \boldsymbol{F})$ such that

$$
p(A)=A\left(A^{*} A\right)^{-1} A^{*} \quad \text { for } \quad A \in V_{n, m}^{\prime}(F) .
$$

The following proposition is well known. 
Proposition 2.5. The above map $p$ induces the smooth embedding

$$
p^{*}: M_{n, m}\left(\boldsymbol{F}^{\prime}\right) \rightarrow H_{m}\left(n ; \boldsymbol{F}^{\prime}\right)
$$

such that $p^{*}$ is a diffeomorphism of $M_{n, m}\left(\boldsymbol{F}^{\prime}\right)$ onto the submanifold $M_{n, m}^{*}(\boldsymbol{F})$ in $H_{m}(n ; \boldsymbol{F})$ and $p=p^{*} \cdot \pi$.

$T\left(V^{\prime}\right), T\left(M^{*}\right)$ will denote the tangent bundles of $V_{n, m}^{\prime}\left(F^{\prime}\right), M_{n, m}^{*}\left(F^{\prime}\right)$ respectively. Let $d p: T\left(V^{\prime}\right) \rightarrow T\left(H_{m}\right)=H_{m}(n ; \boldsymbol{F}) \times H_{0}(n ; \boldsymbol{F})$ be the differential of $p ; V_{n, m}^{\prime}(\boldsymbol{F}) \rightarrow H_{m}(n ; \boldsymbol{F})$, where $T\left(H_{m}\right)$ is the tangent bundle of $H_{m}\left(n ; F^{\prime}\right) . \quad N\left(M^{*}\right)$ will denote the normal bundle of $M_{n, m}^{*}\left(F^{\prime}\right)$ in $H_{m}\left(n ; F^{\prime}\right)$. Then we may consider as follows

$$
\begin{aligned}
& T\left(V^{\prime}\right)=V_{n, m}^{\prime}(\boldsymbol{F}) \times M\left(n, m ; \boldsymbol{F}^{\prime}\right), \\
& T\left(M^{*}\right) \subset M_{n, m}^{*}(\boldsymbol{F}) \times H_{0}\left(n ; \boldsymbol{F}^{\prime}\right), \\
& N\left(M^{*}\right) \subset M_{n, m}^{*}(\boldsymbol{F}) \times H_{0}\left(n ; \boldsymbol{F}^{\prime}\right) .
\end{aligned}
$$

Now we study $d p$ and $T\left(M^{*}\right) . \quad V_{n, m}^{\prime}(\boldsymbol{F})$ is regarded as an open set of $d_{F} m n$-dimensional Euclidean space $M\left(n, m ; F^{\prime}\right)$ and $p$ is the smooth function defined on open set $V_{n, m}^{\prime}\left(F^{\prime}\right)$, valued in the Euclidean space $H(n ; \boldsymbol{F})$. Then we differentiate $p(A)=A\left(A^{*} A\right)^{-1} A^{*}$ and we obtain:

$$
\begin{aligned}
d\left(A\left(A^{*} A\right)^{-1} A^{*}\right)= & \left(E_{n}-A\left(A^{*} A\right)^{-1} A^{*}\right)(d A)\left(A^{*} A\right)^{-1} A^{*} \\
& +A\left(A^{*} A\right)^{-1}\left(d A^{*}\right)\left(E_{n}-A\left(A^{*} A\right)^{-1} A^{*}\right) .
\end{aligned}
$$

This fact shows that $d p$ is as follows:

$$
d p(A, \mathfrak{a})=\left(p(A), \mathfrak{a}_{0}\left(A^{*} A\right)^{-1} A^{*}+A\left(A^{*} A\right)^{-1} \mathfrak{a}_{0}^{*}\right)
$$

where, $A \in V_{n, m}^{\prime}(\boldsymbol{F}), \quad \mathfrak{a} \in M(n, m ; \boldsymbol{F})$ and $\mathfrak{a}_{0}=\left(E_{n}-A\left(A^{*} A\right)^{-1} A^{*}\right) \mathfrak{a}$. The following lemma is easily seen.

LemMA 2.6. For $A \in V_{n, m}^{\prime}(\boldsymbol{F})$ and $\mathfrak{a}_{0} \in M\left(n, m ; F^{\prime}\right), A^{*} a_{0}=0 \in M\left(m ; F^{\prime}\right)$ if and only if there exists an $a \in M(n, m ; \boldsymbol{F})$ such that

$$
\mathfrak{a}_{0}=\left(E_{n}-A\left(A^{*} A\right)^{-1} A^{*}\right) \mathfrak{a} .
$$

Since $\pi ; V_{n, m}^{\prime}(\boldsymbol{F}) \rightarrow M_{n, m}(\boldsymbol{F})$ is the projection of the fiber bundle, $\pi$ is a submmersion. Therefore $p$ is a submmersion of $V_{n, m}^{\prime}\left(\boldsymbol{F}^{\prime}\right)$ onto $M_{n, m}^{*}\left(\boldsymbol{F}^{\prime}\right)$ by Proposition 2.5. Thus $d p: T\left(V^{\prime}\right) \rightarrow T\left(M^{*}\right)$ is surjective. By means of the Gram-Schmidt process, $p\left(V_{n, m}\left(F^{\prime}\right)\right)=M_{n, m}^{*}\left(F^{\prime}\right)$. Therefore we have the following lemma.

LEMMA 2.7.

$$
\begin{aligned}
T\left(M^{*}\right)= & \left\{\left(A\left(A^{*} A\right)^{-1} A^{*}, \mathfrak{a}_{0}\left(A^{*} A\right)^{-1} A^{*}\right.\right. \\
& \left.\left.+A\left(A^{*} A\right)^{-1} \mathfrak{a}_{0}^{*}\right) ; A \in V_{n, m}^{\prime}(\boldsymbol{F}), \mathfrak{a}_{0} \in M(n, m ; \boldsymbol{F}) \text { and } A^{*} \mathfrak{a}_{0}=0\right\} \\
= & \left\{\left(A A^{*}, \mathfrak{a}_{0} A^{*}+A \mathfrak{a}_{0}^{*}\right) ; A \in V_{n, m}(\boldsymbol{F}), \mathfrak{a}_{0} \in M\left(n, m ; \boldsymbol{F}^{\prime}\right) \text { and } A^{*} \mathfrak{a}_{0}=0\right\} .
\end{aligned}
$$


Denote by $T_{P}$ and $N_{P}$ the tangent space and normal space to $M_{n, m}^{*}(F)$ at $P \in M_{n, m}^{*}(F)$, i.e., the fibres of $T\left(M^{*}\right)$ and $N\left(M^{*}\right)$ over $P \in M_{n, m}^{*}\left(F^{\prime}\right)$ respectively. In particular $T_{0}, N_{0}$ denote $T_{P_{0}}, N_{P_{0}}$ respectively, where $P_{0}$ is defined as

$$
P_{0}=\left(\begin{array}{ll}
E_{m} & 0 \\
0 & 0
\end{array}\right) .
$$

By means of Lemma 2.2, Lemma 2.4 and Lemma 2.7, it is not hard to see the following lemma.

LemMa 2.8 .

$$
\begin{aligned}
& T_{0}=\left\{\left(P_{0},\left(\begin{array}{cc}
0 & S^{*} \\
S & 0
\end{array}\right)\right) ; S \in M\left(n-m, m ; \boldsymbol{F}^{\prime}\right)\right\}, \\
& N_{0}=\left\{\left(P_{0},\left(\begin{array}{cc}
K & 0 \\
0 & L
\end{array}\right)\right) ; K \in H(m ; \boldsymbol{F}), L \in H(n-m ; \boldsymbol{F})\right. \\
&\quad \text { and } \operatorname{tr}(K)+\operatorname{tr}(L)=0\} . \\
& T_{P}=\left\{\left(P, U\left(\begin{array}{cc}
0 & S^{*} \\
S & 0
\end{array}\right) U^{*}\right) ; S \in M(n-m, m ; \boldsymbol{F})\right\}, \\
& N_{P}=\left\{\left(P, U\left(\begin{array}{cc}
K & 0 \\
0 & L
\end{array}\right) U^{*}\right) ; K \in H(m ; \boldsymbol{F}), L \in H(n-m ; \boldsymbol{F})\right. \\
&\quad \text { and } \operatorname{tr}(K)+\operatorname{tr}(L)=0\},
\end{aligned}
$$

for $U \in U(n ; \boldsymbol{F})$ such that $P=U P_{0} U^{*}$.

Now to find the second fundamental form (first order normal form) $\nu_{P}$ of $p^{*}$ at $P=p(A) \in M_{n, m}^{*}(\boldsymbol{F})$ for $A \in V_{n, m}^{\prime}(\boldsymbol{F})$, we consider the curve on $V_{n, m}^{\prime}(\boldsymbol{F})$ through $A$, for arbitrary $\mathfrak{a} \in M(n, m ; \boldsymbol{F})$ and sufficiently small $\varepsilon>0$,

$$
\phi_{A}(t)=A+\mathfrak{a} t \text { for }|t|<\varepsilon .
$$

We have the following curve on $M_{n, m}^{*}(\boldsymbol{F})$ through $P$

$$
P_{t}=p\left(\phi_{A}(t)\right) \text { for }|t|<\varepsilon .
$$

Then

$$
\begin{aligned}
\left(\frac{d P_{t}}{d t}\right)_{t=0}= & \mathfrak{a}_{0}\left(A^{*} A\right)^{-1} A^{*}+A\left(A^{*} A\right)^{-1} \mathfrak{a}_{0}^{*}, \\
\left(\frac{d^{2} P_{t}}{d t^{2}}\right)_{t=0}= & 2\left(\mathfrak{a}_{0}\left(A^{*} A\right)^{-1} \mathfrak{a}_{0}^{*}-A\left(A^{*} A\right)^{-1} \mathfrak{a}_{0}^{*} \mathfrak{a}_{0}\left(A^{*} A\right)^{-1} A^{*}\right. \\
& \left.-\mathfrak{a}_{0} X\left(A^{*} A\right)^{-1} A^{*}-A\left(A^{*} A\right)^{-1} X^{*} \mathfrak{a}_{0}^{*}\right),
\end{aligned}
$$


where $\mathfrak{a}_{0}=\left(E_{n}-p(A)\right) \mathfrak{a}, X=\left(A^{*} A\right)^{-1} A^{*}$ a. By Lemma $2.7 \mathfrak{a}_{0} X\left(A^{*} A\right)^{-1} A^{*}+$ $A\left(A^{*} A\right)^{-1} X^{*} \mathfrak{a}_{0}^{*}$ is tangent to $M_{n, m}^{*}(F)$ at $P$. Moreover $\mathfrak{a}_{0}\left(A^{*} A\right)^{-1} \mathfrak{a}_{0}^{*}-$ $A\left(A^{*} A\right)^{-1} \mathfrak{a}_{0}^{*} \mathfrak{a}_{0}\left(A^{*} A\right)^{-1} A^{*}$ is normal to $M_{n, m}^{*}(F)$ at $P$. In fact, for arbitrary $b_{0} \in M(n, m ; F)$ such that $A^{*} b_{0}=0$, set $D_{0}=a_{0}\left(A^{*} A\right)^{-1} A^{*}$ and $D=$ $b_{0}\left(A^{*} A\right)^{-1} A^{*}$, then $\mathfrak{a}_{0}\left(A^{*} A\right)^{-1} \mathfrak{a}_{0}^{*}-A\left(A^{*} A\right)^{-1} \mathfrak{a}_{0}^{*} \mathfrak{a}_{0}\left(A^{*} A\right)^{-1} A^{*}=D_{0} D_{0}^{*}-D_{0}^{*} D_{0}$, $b_{0}\left(A^{*} A\right)^{-1} A^{*}+A\left(A^{*} A\right)^{-1} b_{0}^{*}=D+D^{*} \in T_{P}$, and $D_{0} D=0=D D_{0}$ by Lemma 2.6 and Lemma 2.7. Thus by Lemma 2.1, $\left(D+D^{*}, D_{0} D_{0}^{*}-D_{0}^{*} D_{0}\right)=0$. Thus we have the following lemma.

LEMMA 2.9. The first order normal form $\nu_{P}: T_{P} \rightarrow N_{P}$ of $p^{*}$ at $P \in M_{n, m}^{*}\left(F^{\prime}\right)$ is as follows

$$
\nu_{P}\left(P, D+D^{*}\right)=\left(P, 2\left(D D^{*}-D^{*} D\right)\right)
$$

where $P=p(A), \quad D=\mathfrak{a}_{0}\left(A^{*} A\right)^{-1} A^{*}, \quad A \in V_{n, m}^{\prime}\left(F^{\prime}\right), \quad \mathfrak{a}_{0} \in M(n, m ; \boldsymbol{F}) \quad$ and $A^{*} \mathfrak{a}_{0}=0$.

Now we show that $N_{0}$ is generated by $\nu_{P_{0}}\left(T_{0}\right)$ where

$$
P_{0}=\left(\begin{array}{ll}
E_{m} & 0 \\
0 & 0
\end{array}\right) \text {. }
$$

For $\left(P_{0},\left(\begin{array}{cc}K & 0 \\ 0 & L\end{array}\right)\right) \in N_{0}$, by Lemma 2.3 and Lemma 2.8 there exists $U_{0} \in U(n ; F)$ such that

$$
U_{0}\left(\begin{array}{cccc}
\alpha_{1} & & & 0 \\
& \ddots & & \\
& & \alpha_{m} & \\
& & \alpha_{m+1} \\
& & \ddots & \alpha_{n}
\end{array}\right) U_{0}^{*}=\left(\begin{array}{cc}
K & 0 \\
0 & L
\end{array}\right),
$$

where

$$
U_{0}=\left(\begin{array}{cc}
V & 0 \\
0 & W
\end{array}\right), \quad V \in U\left(m ; \boldsymbol{F}^{\prime}\right), \quad W \in U(n-m ; \boldsymbol{F}), \quad \alpha_{i} \in \boldsymbol{R}(1 \leqq i \leqq n) \text { and }
$$
$\alpha_{1}+\alpha_{2}+\cdots+\alpha_{n}=0$.

Let $E_{i j}$ be a matrix unit of $M(n ; \boldsymbol{F})$ such that the $(i, j)$ component of it is 1 and the other components are 0 . Set

$$
\begin{aligned}
& X_{i}=E_{m+1, m+1}-E_{i, i} \quad \text { for } 1 \leqq i \leqq m, \\
& X_{j}=E_{j+1, j+1}-E_{m, m} \quad \text { for } m+1 \leqq j \leqq n-1, \\
& x_{k}=-\alpha_{k} \text { for } 1 \leqq k<m, \\
& x_{m}=-\left(\alpha_{m}+\alpha_{m+2}+\cdots+\alpha_{n}\right), \\
& x_{k}=\alpha_{k+1} \quad \text { for } m+1 \leqq k \leqq n-1 .
\end{aligned}
$$


Then we have the following

$$
\left(\begin{array}{cc}
K & 0 \\
0 & L
\end{array}\right)=x_{1}\left(U_{0} X_{1} U_{0}^{*}\right)+\cdots+x_{n-1}\left(U_{0} X_{n-1} U_{0}^{*}\right) \text {. }
$$

For $1 \leqq i \leqq m$ and $m+1 \leqq j \leqq n$, set

$$
D=\frac{1}{\sqrt{2}} U_{0} E_{j i} U_{0}^{*}
$$

Then

$$
\left(P_{0}, D+D^{*}\right) \in T_{0}, \quad 2\left(D D^{*}-D^{*} D\right)=U_{0}\left(E_{j j}-E_{i i}\right) U_{0}^{*} .
$$

Thus $\left(P_{0}, U_{0} X_{i} U_{0}^{*}\right) \in \nu_{P_{0}}\left(T_{0}\right)$ for $1 \leqq i \leqq n-1$, i.e., $N_{0}$ is generated by $\nu_{P_{0}}\left(T_{0}\right)$. By Lemma 2.8, Theorem 2.9 and the above facts, we obtain the following main result.

THEOREM 2.10. $p^{*}: M_{n, m}(\boldsymbol{F}) \rightarrow H_{m}\left(n ; \boldsymbol{F}^{\prime}\right)$ is an inflection-free embedding.

3. Tangent bundles of Grassmann manifolds. Let $T(V)$ be the tangent bundle of Stiefel manifold $V_{n, m}(\boldsymbol{F})$. It is not hard to see the following

$$
T(V)=\left\{(A, \mathfrak{a}) \in V_{n, m}\left(\boldsymbol{F}^{\prime}\right) \times M(n, m ; \boldsymbol{F}) ; A^{*} \mathfrak{a}+\mathfrak{a}^{*} A=0\right\} .
$$

Set

$$
\begin{aligned}
& T_{0}\left(V_{n, m}^{\prime}\left(\boldsymbol{F}^{\prime}\right)\right)=T_{0}\left(V^{\prime}\right)=\left\{(A, \mathfrak{a}) \in V_{n, m}^{\prime}\left(\boldsymbol{F}^{\prime}\right) \times M\left(n, m ; \boldsymbol{F}^{\prime}\right) ; A^{*} \mathfrak{a}=0\right\}, \\
& T_{0}\left(V_{n, m}\left(\boldsymbol{F}^{\prime}\right)\right)=T_{0}(V)=\left\{(A, \mathfrak{a}) \in V_{n, m}\left(\boldsymbol{F}^{\prime}\right) \times M\left(n, m ; \boldsymbol{F}^{\prime}\right) ; A^{*} \mathfrak{a}=0\right\} .
\end{aligned}
$$

Then $T_{0}\left(V^{\prime}\right), T_{0}(V)$ are subbundles of $T\left(V^{\prime}\right), T(V)$ respectively.

For $(A, \mathfrak{a}),(B, \mathfrak{b}) \in T_{0}\left(V^{\prime}\right)\left(\right.$ resp. $\left.T_{0}(V)\right)$, suppose that $d p(A, \mathfrak{a})=d p(B, \mathfrak{b})$ then there exists $g \in G L\left(m ; F^{\prime}\right)$ (resp. $U\left(m ; F^{\prime}\right)$ ) such that $B=A g$ and $\mathfrak{b}=a g$. In fact by $d p(A, \mathfrak{a})=d p(B, \mathfrak{b}), B\left(B^{*} B\right)^{-1} B^{*}=A\left(A^{*} A\right)^{-1} A^{*}$ and

$$
\mathfrak{b}\left(B^{*} B\right)^{-1} B^{*}+B\left(B^{*} B\right)^{-1} \mathfrak{b}^{*}=\mathfrak{a}\left(A^{*} A\right)^{-1} A^{*}+A\left(A^{*} A\right)^{-1} \mathfrak{a}^{*} \text {. }
$$

Thus $B=A\left(A^{*} A\right)^{-1} A^{*} B, \mathfrak{b}=\mathfrak{a}\left(A^{*} A\right)^{-1} A^{*} B$ and $B^{*} B=\left(A^{*} B\right)^{*}\left(A^{*} A\right)^{-1} A^{*} B$. By Lemma $2.7 d p$ is a surjection of $T_{0}\left(V^{\prime}\right)$ (resp. $T_{0}(V)$ ) to $T\left(M^{*}\right)$ covering $p: V_{n, m}^{\prime}\left(\boldsymbol{F}^{\prime}\right) \rightarrow M_{n, m}^{*}\left(\boldsymbol{F}^{\prime}\right)$ (resp. $V_{n, m}\left(\boldsymbol{F}^{\prime}\right) \rightarrow M_{n, m}^{*}\left(\boldsymbol{F}^{\prime}\right)$ ). Now $G L\left(m ; \boldsymbol{F}^{\prime}\right)$ (resp. $U\left(m ; F^{\prime}\right)$ ) acts on $T_{0}\left(V^{\prime}\right)$ and $V_{n, m}^{\prime}\left(F^{\prime}\right)$ (resp. $T_{0}(V)$ and $V_{n, m}\left(F^{\prime}\right)$ ) on the right as follows, for $((A, \mathfrak{a}), g) \in T_{0}\left(V^{\prime}\right) \times G L\left(m ; F^{\prime}\right)$ (resp. $T_{0}(V) \times$ $U(m ; \boldsymbol{F})), \quad((A, \mathfrak{a}), g) \rightarrow(A g, \mathfrak{a} g) \in T_{0}\left(V^{\prime}\right)$ (resp. $\left.T_{0}(V)\right)$. It is not hard to see that $T_{0}\left(V^{\prime}\right)$ (resp. $\left.T_{0}(V)\right)$ is a real right $G L\left(m ; \boldsymbol{F}^{\prime}\right)$-(resp. $U\left(m ; \boldsymbol{F}^{\prime}\right)$-) vector bundle over right $G L\left(m ; \boldsymbol{F}^{\prime}\right)$-(resp. $U\left(m ; \boldsymbol{F}^{\prime}\right)$-) space $V_{n, m}^{\prime}\left(\boldsymbol{F}^{\prime}\right)$ (resp. $V_{n, m}\left(\boldsymbol{F}^{\prime}\right)$ ). In particular $T_{0}\left(V_{n, m}^{\prime}(\boldsymbol{C})\right)$ (resp. $T_{0}\left(V_{n, m}(\boldsymbol{C})\right)$ is a complex right $G L(m ; C)$-(resp. $U(m)-)$ vector bundle over right $G L(m ; C)$-(resp. $U(m)-)$ space $V_{n, m}^{\prime}(C)$ (resp. $V_{n, m}(C)$ ). Therefore the orbit space 


\section{$T_{0}\left(V^{\prime}\right) / G L\left(m ; \boldsymbol{F}^{\prime}\right)\left(\operatorname{resp} . T_{0}(V) / U\left(m ; \boldsymbol{F}^{\prime}\right)\right)$}

may be regarded as a real vector bundle over $M_{n, m}\left(F^{\prime}\right)$. In particular $T_{0}\left(V_{n, m}^{\prime}(\boldsymbol{C})\right) / G L(m ; C)\left(\operatorname{resp} . T_{0}\left(V_{n, m}(C)\right) / U(m)\right)$ may be regarded as a complex vector bundle over $M_{n, m}(C)$. By the facts stated above, we have the following theorem.

THEOREM 3.1. $\quad d p: T_{0}\left(V^{\prime}\right) \rightarrow T\left(M^{*}\right) \quad\left(\right.$ resp. $\left.T_{0}(V) \rightarrow T\left(M^{*}\right)\right)$ induces a bundle equivalence map $d p^{*}: T_{0}\left(V_{n, m}^{\prime}\left(\boldsymbol{F}^{\prime}\right)\right) / G L(m ; \boldsymbol{F}) \rightarrow T\left(M_{n, m}^{*}(\boldsymbol{F})\right) \quad$ (resp. $\left.T_{0}\left(V_{n, m}\left(\boldsymbol{F}^{\prime}\right)\right) / U\left(m ; \boldsymbol{F}^{\prime}\right) \rightarrow T\left(M_{n, m}^{*}\left(\boldsymbol{F}^{\prime}\right)\right)\right)$ covering $p^{*}: M_{n, m}\left(\boldsymbol{F}^{\prime}\right) \rightarrow M_{n, m}^{*}\left(\boldsymbol{F}^{\prime}\right)$ as real vector bundle, where $T\left(M_{n, m}^{*}\left(F^{\prime}\right)\right)=T\left(M^{*}\right)$.

Thus we may identify $T_{0}\left(V_{n, m}^{\prime}\left(\boldsymbol{F}^{\prime}\right)\right) / G L\left(m ; \boldsymbol{F}^{\prime}\right)$ with the tangent bundle $T\left(M_{n, m}\left(F^{\prime}\right)\right)$ of $M_{n, m}\left(F^{\prime}\right)$.

$T_{0}\left(V_{n, m}^{\prime}(C)\right) / G L(m ; C)$ is a complex vector bundle over $M_{n, m}(\boldsymbol{C})$. $T\left(M_{n, m}(C)\right)$ has complex structure. It is easily seen that $T_{0}\left(V_{n, m}^{\prime}(C)\right) / G L(m ; C)$ may be identified with $T\left(M_{n, m}(C)\right)$ as complex vector bundle. For the rest of this paper, we regard $T\left(M_{n, m}(C)\right)$ as a complex vector bundle.

The fibre's dimension of right $F$-vector bundle $T_{0}\left(V^{\prime}\right)\left(T_{0}(V)\right)$ is $m(n-m)$. Moreover we have the following short exact sequences (i), (ii) of vector bundles over $V_{n, m}^{\prime}\left(\boldsymbol{F}^{\prime}\right), V_{n, m}\left(\boldsymbol{F}^{\prime}\right)$ respectively

$$
\begin{aligned}
& 0 \longrightarrow V_{n, m}^{\prime}\left(\boldsymbol{F}^{\prime}\right) \times M\left(m ; \boldsymbol{F}^{\prime}\right) \stackrel{\phi}{\longrightarrow} T\left(V^{\prime}\right) \stackrel{\psi}{\longrightarrow} T_{0}\left(V^{\prime}\right) \longrightarrow 0 \\
& 0 \longrightarrow V_{n, m}(\boldsymbol{F}) \times \mathfrak{U}(m ; \boldsymbol{F}) \stackrel{\phi}{\longrightarrow} T(V) \stackrel{\psi}{\longrightarrow} T_{0}(V) \longrightarrow 0
\end{aligned}
$$

where $\phi$ and $\psi$ of (i) are defined by

$$
\begin{aligned}
& \phi(A, X)=(A, A X) \quad \text { for } \quad(A, X) \in V_{n, m}^{\prime}(\boldsymbol{F}) \times M(m ; \boldsymbol{F}), \\
& \psi(A, \mathfrak{a})=\left(A,\left(E_{n}-A\left(A^{*} A\right)^{-1} A^{*}\right) \mathfrak{a}\right) \text { for }(A, \mathfrak{a}) \in T\left(V^{\prime}\right),
\end{aligned}
$$

and $\mathfrak{U}(m ; \boldsymbol{F})=\left\{X \in M(m ; \boldsymbol{F}) ; X^{*}+X=0\right\}, \phi$ and $\psi$ of (ii) are the restrictions of them of (i) respectively. Now (i), (ii) have natural splitting homomorphisms $\quad \omega: T\left(V^{\prime}\right) \rightarrow V_{n, m}^{\prime}(\boldsymbol{F}) \times M(m ; \boldsymbol{F}), \quad \omega: T(V) \rightarrow V_{n, m}(\boldsymbol{F}) \times$ $\mathfrak{u}(m ; \boldsymbol{F})$ defined by

$$
\omega(A, \mathfrak{a})=\left(A,\left(A^{*} A\right)^{-1} A^{*} \mathfrak{a}\right) \quad \text { for } \quad(A, \mathfrak{a}) \in T\left(V^{\prime}\right), T(V) .
$$

Then $\phi, \psi$ and $\omega$ of (i) are right $\boldsymbol{F}$-vector bundle homomorphisms, but them of (ii) are real vector bundle homomorphisms.

Now let $V, W$ be right $\boldsymbol{F}$-vector spaces of dimension $m, n$ respectively. $L_{F}(V, W)$ denotes set of all $\boldsymbol{F}$-homomorphisms from $V$ to $W$. It is a vector space over $\boldsymbol{R}$ if $\boldsymbol{F}=\boldsymbol{R}$ or $\boldsymbol{H}$, over $\boldsymbol{C}$ if $\boldsymbol{F}=\boldsymbol{C}$.

Let $\eta, \zeta$ be right $F$-vector bundles over compact manifold $M$. It is 
possible to define in natural way the vector bundle $L_{F}(\eta, \zeta)$ whose fibre $L_{F}(\eta, \zeta)_{x}$ at $x \in M$ is $L_{F}\left(\eta_{x}, \zeta_{x}\right)$, where $\eta_{x}, \zeta_{x}$ are fibres of $\eta$, $\zeta$ at $x$ respectively. It is a real vector bundle if $\boldsymbol{F}=\boldsymbol{R}$ or $\boldsymbol{H}$, a complex vector bundle if $\boldsymbol{F}=\boldsymbol{C}$.

$\xi$ denotes the canonical right $\boldsymbol{F}$-vector bundle over $M_{n, m}(\boldsymbol{F})$, i.e., its total space $E_{\xi}$ is as follows

$$
E_{\xi}=\left\{(V, \boldsymbol{x}) \in M_{n, m}(\boldsymbol{F}) \times \boldsymbol{F}^{n} ; \boldsymbol{x} \in V\right\} .
$$

Let $\xi^{\perp}$ be orthogonal complement of $\xi$ in $M_{n, m}\left(F^{\prime}\right) \times F^{n}$, i.e.,

$$
E_{\xi^{\perp}}=\left\{(V, \boldsymbol{x}) \in M_{n, m}(\boldsymbol{F}) \times \boldsymbol{F}^{n} ; \boldsymbol{x} \in V^{\perp}\right\},
$$

where $V^{\perp}$ is orthogonal complement of $V$ in $\boldsymbol{F}^{n}$. We have the following short exact sequence of right $\boldsymbol{F}$-vector bundles over $M_{n, m}(\boldsymbol{F})$.

$$
0 \longrightarrow \xi \stackrel{i}{\longrightarrow} M_{n, m}(\boldsymbol{F}) \times \boldsymbol{F}^{n} \stackrel{j}{\longrightarrow} \xi^{\perp} \longrightarrow 0
$$

where $i$ is inclusion map and $j$ is as follows for $(V, \boldsymbol{x}) \in M_{n, m}(\boldsymbol{F}) \times \boldsymbol{F}^{n}$, there exists $A \in V_{n, m}^{\prime}(\boldsymbol{F})$ such that $m$ column vectors of $A$ span $V$ and

$$
j(V, \boldsymbol{x})=\left(V,\left(E_{n}-A\left(A^{*} A\right)^{-1} A^{*}\right) \boldsymbol{x}\right) .
$$

The above exact sequence has canonical splitting $\boldsymbol{F}$-homomorphism. It is as follows $q ; M_{n, m}(\boldsymbol{F}) \times \boldsymbol{F}^{n} \rightarrow \xi$

$$
q(V, \boldsymbol{x})=\left(V, A\left(A^{*} A\right)^{-1} A^{*} \boldsymbol{x}\right) \quad \text { for } \quad(V, \boldsymbol{x}) \in M_{n, m}(\boldsymbol{F}) \times \boldsymbol{F}^{n} .
$$

where $m$ column vectors of $A \in V_{n, m}^{\prime}(\boldsymbol{F})$ span $V$. Then we have the following short exact sequence of vector bundles over $M_{n, m}\left(F^{\prime}\right)$.

$$
0 \longrightarrow L_{F}(\xi, \xi) \stackrel{i_{*}}{\longrightarrow} L_{F}\left(\xi, F^{n}\right) \stackrel{j_{*}}{\longrightarrow} L_{F}\left(\xi, \xi^{\perp}\right) \longrightarrow 0
$$

where $L_{F}\left(\xi, F^{n}\right)=L_{F}\left(\xi, M_{n, m}\left(F^{\prime}\right) \times F^{n}\right)$. (iii) has canonical splitting homomorphism $q_{*}: L_{F}\left(\xi, F^{n}\right) \rightarrow L_{F}(\xi, \xi)$ or $\iota_{*}: L_{F}\left(\xi, \xi^{\perp}\right) \rightarrow L_{F}\left(\xi, F^{n}\right)$, where ८: $\xi^{\perp} \rightarrow M_{n, m}\left(\boldsymbol{F}^{\prime}\right) \times \boldsymbol{F}^{n}$ inclusion map.

In general, let $V, W$ be right $\boldsymbol{F}$-vector spaces of dimension $m, n$ respectively and let $f$ be a $F$-linear map from $V$ to $W$. Suppose that $\left\{\boldsymbol{a}_{1}, \cdots, \boldsymbol{a}_{m}\right\}, \quad\left\{\boldsymbol{a}_{1}^{\prime}, \cdots, \boldsymbol{a}_{m}^{\prime}\right\}$ are two bases of $V$ such that $\left\{\boldsymbol{a}_{1}^{\prime} \cdots, \boldsymbol{a}_{m}^{\prime}\right\}=$ $\left\{\boldsymbol{a}_{1}, \cdots, \boldsymbol{a}_{m}\right\} g$, where $g \in G L(m ; \boldsymbol{F})$, that $\left\{\boldsymbol{b}_{1}, \cdots, \boldsymbol{b}_{n}\right\},\left\{\boldsymbol{b}_{1}^{\prime}, \cdots, \boldsymbol{b}_{n}^{\prime}\right\}$ are two bases of $W$ such that $\left\{\boldsymbol{b}_{1}^{\prime}, \cdots, \boldsymbol{b}_{n}^{\prime}\right\}=\left\{\boldsymbol{b}_{1}, \cdots, \boldsymbol{b}_{n}\right\} h$, where $h \in G L(n ; \boldsymbol{F})$, that $X \in M(n, m ; \boldsymbol{F})$ corresponds to $f$ with respect to $\left\{\boldsymbol{a}_{1}, \cdots, \boldsymbol{a}_{m}\right\},\left\{\boldsymbol{b}_{1}, \cdots, \boldsymbol{b}_{n}\right\}$ and that $X^{\prime} \in M(n, m ; \boldsymbol{F})$ corresponds to $f$ with respect to $\left\{\boldsymbol{a}_{1}^{\prime}, \cdots, \boldsymbol{a}_{m}^{\prime}\right\}$, $\left\{\boldsymbol{b}_{1}^{\prime}, \cdots, \boldsymbol{b}_{n}^{\prime}\right\}$, then $X^{\prime}=h^{-1} X g$.

By this fact we have the following commutative diagram 
(iv)

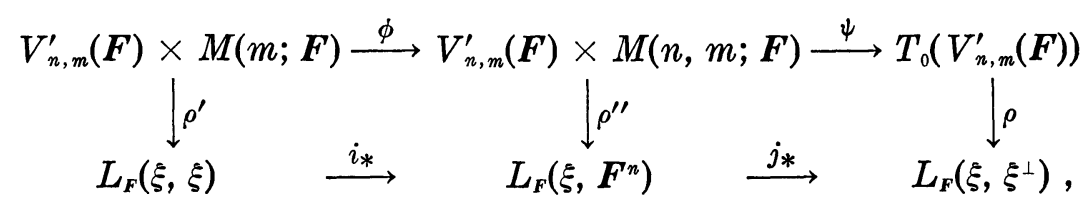

where $\rho^{\prime}, \rho^{\prime \prime}$ and $\rho$ are surjective bundle maps covering $\pi: V_{n, m}^{\prime}(\boldsymbol{F}) \rightarrow$ $M_{n, m}(\boldsymbol{F})$.

Now $G L(m ; \boldsymbol{F})$ acts on $V_{n, m}^{\prime}(\boldsymbol{F}) \times M(m ; \boldsymbol{F}), V_{n, m}^{\prime}(\boldsymbol{F}) \times M(n, m ; \boldsymbol{F})$ and $T_{0}\left(V_{n, m}^{\prime}(F)\right)$ on the right as follows

( $(A, X), g) \times\left(A g, g^{-1} X g\right) \quad$ for $\quad A \in V_{n, m}^{\prime}(\boldsymbol{F}), X \in M(m ; \boldsymbol{F}), g \in G L\left(m ; \boldsymbol{F}^{\prime}\right)$, $((A, \mathfrak{a}), g) \rightarrow(A g, \mathfrak{a} g)$ for $\quad A \in V_{n, m}^{\prime}(\boldsymbol{F}), \mathfrak{a} \in M(n, m ; \boldsymbol{F}), g \in G L(m ; \boldsymbol{F})$.

It is not hard to see that $\rho^{\prime}, \rho^{\prime \prime}$ and $\rho$ induce bundle equivalence maps $\rho_{*}^{\prime}, \rho_{*}^{\prime \prime}$ and $\rho_{*}$ covering $\pi^{*}: V_{n, m}^{\prime}(\boldsymbol{F}) / G L(m ; \boldsymbol{F}) \rightarrow M_{n, m}(\boldsymbol{F})$ as follows

(v)
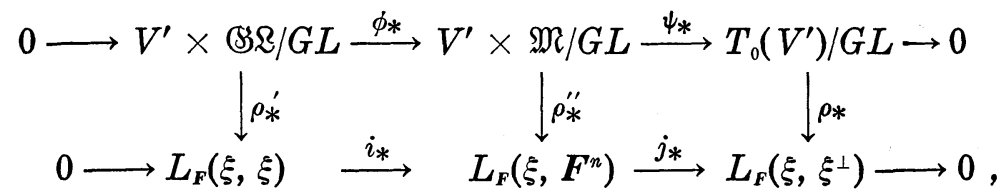

where $\quad V^{\prime}=V_{n, m}^{\prime}\left(\boldsymbol{F}^{\prime}\right), \quad \mathbb{S} \mathbb{R}=M(m ; \boldsymbol{F}), \quad G L(m ; \boldsymbol{F}), \quad \mathfrak{M}=M(n, m ; \boldsymbol{F}), \quad$ all horizontal sequences are exact and this diagram is commutative. By Theorem 3.1 we have the following theorem.

ThEOREM 3.2. (Milnor [6]) Tangent bundle of $M_{n, m}(\boldsymbol{F})$ is isomorphic to $L_{F}\left(\xi, \xi^{\perp}\right)$.

It is easy to see that

$$
L_{\boldsymbol{F}}\left(\xi, \boldsymbol{F}^{n}\right)=L_{\boldsymbol{F}}(\xi, \boldsymbol{F}) \oplus \cdots \oplus L_{\boldsymbol{F}}(\xi, \boldsymbol{F})=n L_{\boldsymbol{F}}(\xi, \boldsymbol{F}),
$$

where $n L_{F}(\xi, \boldsymbol{F})$ denotes the $n$-fold Whitney sum of $L_{F}(\xi, \boldsymbol{F})$ and $\boldsymbol{F}$ is trivial right $\boldsymbol{F}$-line bundle over $M_{n, m}(\boldsymbol{F})$. We have the following theorem.

Theorem 3.3. (W. C. Hsiang and R. H. Szczarba [4])

$$
T\left(M_{n, m}(\boldsymbol{F})\right) \oplus L_{\boldsymbol{F}}(\xi, \xi)=n L_{F}(\xi, \boldsymbol{F}) .
$$

$\xi$ may be regarded as a right $\boldsymbol{F}$-vector bundle with structure group $U(m ; \boldsymbol{F})$ which acts on its fibre on the left. Then $L_{\boldsymbol{R}}(\xi, \boldsymbol{R})=\xi$ and $L_{R}(\xi, \xi)=\xi \bigotimes_{R} \xi$. Thus we have the corollary

CoROLlary 3.4. $T\left(M_{n, m}(R)\right) \oplus \xi \otimes_{\mathrm{R}} \xi=n \xi$.

$L_{c}(\xi, C)=\bar{\xi}$, where $\bar{\xi}$ denotes complex conjugate bundle of $\xi$ and $L_{c}(\xi, \xi)=\bar{\xi} \otimes_{c} \xi$ (see 3.13 lemma and 3.17 corollary in [1]). We denote the complex tangent bundle of $M_{n, m}(C)$ by $T\left(M_{n, m}(C)\right)$. We have the 
following corollary.

Corollary 3.5. $T\left(M_{n, m}(C)\right) \oplus \bar{\xi} \bigotimes_{c} \xi=n \bar{\xi}$.

Now we will show that $L_{H}(\xi, H)=\xi_{R}$, where $\xi_{R}$ denotes the real vector bundle defined by $\xi$. Let $G$ be a group and let $V$ be a $m$-dimensional $G$-vector space over $\boldsymbol{H}$ which is a right $\boldsymbol{H}$-vector space such that $G$ acts on it on the left. Then we can regard the dual space $L_{H}(V, \boldsymbol{H})=V^{*}$ as a right $\boldsymbol{H}$-vector space on which $G$ acts on the left as follows, for $\phi \in V^{*}, q \in \boldsymbol{H}$ and $g \in G,(g \phi q)(v)=\bar{q}\left(\phi\left(g^{-1} v\right)\right)$ for $v \in V$. Let $\left\{\boldsymbol{e}_{1}, \cdots, \boldsymbol{e}_{m}\right\}$ be a basis of $V$ and let $\left\{\delta_{1}, \cdots, \delta_{m}\right\}$ be the dual basis of $V^{*}$ with respect to $\left\{\boldsymbol{e}_{1}, \cdots, \boldsymbol{e}_{m}\right\}$. For $\boldsymbol{v}=\boldsymbol{e}_{1} x_{1}+\cdots+\boldsymbol{e}_{m} x_{m} \in V, \phi=$ $\delta_{1} \lambda_{1}+\cdots+\delta_{m} \lambda_{m} \in V^{*}$ and $g \in G$ let

$$
g \boldsymbol{v}=\left(\boldsymbol{e}_{1}, \cdots, \boldsymbol{e}_{m}\right) X_{g}\left(\begin{array}{c}
x_{1} \\
\vdots \\
x_{m}
\end{array}\right), \quad g \phi=\left(\delta_{1}, \cdots, \delta_{m}\right)\left(\begin{array}{c}
\mu_{1} \\
\vdots \\
\mu_{m}
\end{array}\right)=\left(\delta_{1}, \cdots, \delta_{m}\right) Y_{g}\left(\begin{array}{c}
\lambda_{1} \\
\vdots \\
\lambda_{m}
\end{array}\right) \text {, }
$$

where $X_{g}, Y_{g} \in G L(m ; \boldsymbol{F})$ and $\left(\begin{array}{c}\mu_{1} \\ \vdots \\ \mu_{m}\end{array}\right)=Y_{g}\left(\begin{array}{c}\lambda_{1} \\ \vdots \\ \lambda_{m}\end{array}\right)$.

Then for $v \in V$

$$
\begin{aligned}
(g \phi)(\boldsymbol{v}) & =\bar{\lambda}_{1} \phi\left(g^{-1} \boldsymbol{v}\right)+\cdots+\bar{\lambda}_{m} \phi\left(g^{-1} \boldsymbol{v}\right)=\left(\bar{\lambda}_{1}, \cdots, \bar{\lambda}_{m}\right) X_{g-1}\left(\begin{array}{c}
x_{1} \\
\vdots \\
x_{m}
\end{array}\right) \\
& =\left(\bar{\mu}_{1}, \cdots, \bar{\mu}_{m}\right)\left(\begin{array}{c}
\delta_{1}(\boldsymbol{v}) \\
\vdots \\
\delta_{m}(\boldsymbol{v})
\end{array}\right)=\left(\bar{\lambda}_{1}, \cdots, \bar{\lambda}_{m}\right) Y_{g}^{*}\left(\begin{array}{c}
x_{1} \\
\vdots \\
x_{m}
\end{array}\right) .
\end{aligned}
$$

Thus $Y_{g}^{*}=X_{g-1}$, i.e., $Y_{g}=\left(X_{g}^{-1}\right)^{*}$.

Now let $\left\{g_{j i}\right\}$ be set of transition functions of the vector bundle $\xi$ with structure group $S p(m)$. Then by the above fact, the set $\left\{h_{j i}\right\}$ of transition functions of vector bundle $L_{H}(\xi, \boldsymbol{H})$ is as follows for each $h_{j i}$

$$
h_{j i}(x)=\left(\left(g_{j i}(x)\right)^{-1}\right)^{*}=g_{j i}(x) \text { for } x \in U_{i} \cap U_{j},
$$

where $\left\{U_{i}\right\}$ is open covering of base space $M_{n, m}(\boldsymbol{H})$ of $\xi$ such that each $g_{j i}: U_{i} \cap U_{j} \rightarrow S p(m)$. Therefore $L_{H}(\xi, \boldsymbol{H})=\xi_{R}$, since we regard $L_{H}(\xi, \boldsymbol{H})$ as a real vector bundle. Thus we have the following corollary.

Corollary 3.6. $T\left(M_{n, m}(\boldsymbol{H})\right) \oplus L_{H}(\xi, \xi)=n \xi_{R}$.

Now $\eta, \zeta$ denote right $\boldsymbol{H}$-line bundles over a space $B$ with structure group $S p(1)=S^{3}$ which acts on their fibres on the left. We derive the 
H. ÔIKE

Pontrjagin class of real vector bundle $L_{H}(\eta, \zeta)$ whose fibre is of dimension 4. Let $V, W$ be right $\boldsymbol{H}$-vector spaces of dimension $m, n$ respectively. $c_{H} V, c_{H} W$ denote complex vector spaces defined by restricting the scalars to $\boldsymbol{C}$ from right $\boldsymbol{H}$-vector spaces $V, W$ respectively and $c L_{H}(V, W)$ denotes $4 m n$-dimensional complex vector space obtained by complexifying the $4 m n$-dimensional real vector space $L_{H}(V, W)$. The following lemma is described in p. 32 of [1].

Lemma 3.7. $c L_{H}(V, W)=L_{c}\left(c_{H} V, c_{H} W\right)$.

Moreover let $G$ be a compact Lie group and suppose that $V, W$ are left $G$-vector spaces then by 3.6 Proposition, 3.13 Lemma, 3.17 Corollary in [1] and the above Lemma 3.7, we have the following lemma.

LemMA 3.8. $c L_{H}(V, W)=c_{H} V \otimes_{c} c_{H} W$.

CoRollaRY 3.9. In Corollary 3.6, $c \xi_{R}=2 c_{H} \xi$ and $c L_{H}(\xi, \xi)=$ $c_{H} \xi \bigotimes_{C} c_{H} \xi$.

$e(\eta), e(\zeta)$ denote the total symplectic Pontrjagin classes of $\eta, \zeta$ respectively and $e_{1}(\eta)=y, e_{1}(\zeta)=z \in H^{4}(B ; Z)$ denote the first symplectic Pontrjagin classes of $\eta, \zeta$ respectively (see 9.6 in [2]). We prove the following proposition.

Proposition 3.10. The total Pontrjagin class of $L_{H}(\eta, \zeta)$ is as follows

$$
p\left(L_{H}(\eta, \zeta)\right)=1+2(y+z)+(y-z)^{2},
$$

i.e., the first Pontrjagin class and the second Pontrjagin class of $L_{H}(\eta, \zeta)$ are as follow

$$
p_{1}\left(L_{H}(\eta, \zeta)\right)=2(y+z), \quad p_{2}\left(L_{H}(\eta, \zeta)\right)=(y-z)^{2} .
$$

Proof. We denote formal factorizations of $e(\eta), e(\zeta)$ by $e(\eta)=1+\alpha^{2}$, $e(\zeta)=1+\beta^{2}$ respectively, where $\alpha^{2}=y, \beta^{2}=z$. These formulas are equivalent to the following total Chern classes of complex vector bundle $c_{H} \eta, c_{H} \zeta$ (see 9.6 in [2])

$$
C\left(c_{H} \eta\right)=(1-\alpha)(1+\alpha), \quad C\left(c_{H} \zeta\right)=(1-\beta)(1+\beta) .
$$

Thus

$$
\begin{aligned}
C\left(c_{H} \eta \otimes_{C} c_{H} \zeta\right) & =(1-\alpha-\beta)(1+\alpha-\beta)(1-\alpha+\beta)(1+\alpha+\beta) \\
& =\left(1-(\alpha+\beta)^{2}\right)\left(1-(\alpha-\beta)^{2}\right) \\
& =1-2\left(\alpha^{2}+\beta^{2}\right)+\left(\alpha^{2}-\beta^{2}\right)^{2} .
\end{aligned}
$$

By Lemma 3.8 we have the following total Chern class of complex vector bundle $c L_{H}(\eta, \zeta)$ 


$$
C\left(c L_{H}(\eta, \zeta)\right)=1-2(y+z)+(y-z)^{2} .
$$

Thus

$$
p\left(L_{H}(\eta, \zeta)\right)=1+2(y+z)+(y-z)^{2} .
$$

For example let $\xi$ be canonical quaternionic line bundle over quaternion projective space $P_{n-1}(\boldsymbol{H})=M_{n, 1}(\boldsymbol{H})$ and let $u \in H^{4}\left(P_{n-1}(\boldsymbol{H}) ; Z\right)$ be the first symplectic Pontrjagin class of $\xi$. Then by Proposition 3.9 we have

$$
p\left(L_{H}(\xi, \xi)\right)=1+4 u .
$$

$\xi \bigotimes_{H} \xi^{*}$ in [4] may be regarded as $L_{H}(\xi, \xi)$. By Lemma 4.1 in p. 703 of [4], $p\left(\xi \otimes_{H} \xi^{*}\right)=1+2 u$. The author thinks that the Lemma 4.1 must be as follows

$$
\begin{aligned}
& p_{1}\left(\xi \bigotimes_{H} \zeta^{*}\right)=2\left(p_{1}(\xi)+p_{1}(\zeta)\right), \\
& p_{2}\left(\xi \bigotimes_{H} \zeta^{*}\right)=\left[p_{1}(\xi)-p_{1}(\zeta)\right]^{2} .
\end{aligned}
$$

Let $\eta, \zeta$ be right $\boldsymbol{H}$-vector bundles over a space $B$ with structure groups $S p(m), S p(n)$ which act on their fibres on the left respectively and let total symplectic Pontrjagin classes of $\eta, \zeta$ and their formal factorizations be as follows

$$
\begin{aligned}
& e(\eta)=1+e_{1}(\eta)+\cdots+e_{m}(\eta)=\left(1+y_{1}\right) \cdots\left(1+y_{m}\right), \\
& e(\zeta)=1+e_{1}(\zeta)+\cdots+e_{n}(\zeta)=\left(1+z_{1}\right) \cdots\left(1+z_{n}\right) .
\end{aligned}
$$

Then proposition in p. 703 of [4] is as follows.

Proposition 3.11. The total Pontrjagin class of $L_{H}(\eta, \zeta)$ is given by

$$
p\left(L_{H}(\eta, \zeta)\right)=\prod_{i=1}^{m} \prod_{j=1}^{n}\left[1+2\left(y_{i}+z_{j}\right)+\left(y_{i}-z_{j}\right)^{2}\right] .
$$

This proposition is an immediate consequence of the splitting principal and Proposition 3.10.

\section{REFERENCES}

[1] J. F. AdAMS, Lectures on Lie Groups, Benjamin, 1969.

[2] A. Borel aNd F. Hirzebruch, Characteristic classes and homogeneous spaces I, Amer. J. Math., 80 (1958), 458-538.

[3] E. A. Feldman, The geometry of immersions I, Trans. Amer. Math. Soc., 120 (1965), 185-224.

[4] W. C. Hsiang and R. H. Szczarba, On the tangent bundle of a Grassman manifold, Amer. J. Math., 86 (1964), 698-704. 
[5] J. A. Little, On singularities submanifolds of higher dimensional Euclidean spaces, Annali di Mat. pura ed appl. (IV), 83 (1969), 261-336.

[6] J. MilnoR, Characteristic classes, (mimeographed notes), January 1962.

[7] H. ÔIKE, Higher order tangent bundles projective spaces lens spaces, Tôhoku Math. J., 22 (1970), 200-209.

[8] W. F. PoHL, Differential geometry of higher order, Topology 1 (1962), 162-211.

[9] H. Suzukr, Bounds for dimensions of odd order non-singular immersions of $R P^{n}$, Trans. Amer. Math. Soc. 121 (1966), 269-275.

[10] H. SuzukI, Characteristic classes of some higher order tangent bundles of complex projective spaces, J. Math. Soc. Japan 18 (1966), 386-393.

[11] H. SuzukI, Higher order non-singular immersions in projective spaces, Quart. J. Math. Oxford (2) 20 (1969), 33-44.

[12] H. SuzukI, On higher order non-singular immersions of $R P^{n}$ in $C P^{m}$, J. Fac. Sci. Hokkaido Univ. (Ser. 1) 22 (1972), 161-170.

[13] I. YoкотA, Group and topology, (in Japanese), Shôkabô (Tokyo), 1971.

[14] C. Yoshioka, On the higher order non-singular immersion, Sci. Rep. Niigata Univ. (Ser. A) 5 (1967), 23-30.

Department OF MATHEMATICS

YAMAGATA UNIVERSITY

990 YamaGatA, JAPAN 